\title{
Improving the Convergence Period of Adaptive Data Rate in a Long Range Wide Area Network for the Internet of Things Devices
}

\author{
Khola Anwar ${ }^{1}$, Taj Rahman 1,*, Asim Zeb ${ }^{2, *}$, Yousaf Saeed ${ }^{3}$, Muhammad Adnan Khan 4,5,*D, Inayat Khan ${ }^{6}$,

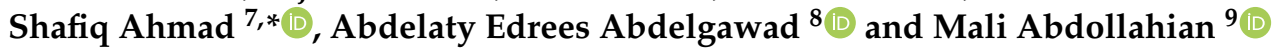

Citation: Anwar, K.; Rahman, T.; Zeb, A.; Saeed, Y.; Khan, M.A.; Khan, I.; Ahmad, S.; Abdelgawad, A.E.; Abdollahian, M. Improving the Convergence Period of Adaptive Data Rate in a Long Range Wide Area Network for the Internet of Things Devices. Energies 2021, 14 5614. https://doi.org/10.3390/ en14185614

Academic Editors: Chang Wu Yu and Naveen Chilamkurti

Received: 6 July 2021

Accepted: 31 August 2021

Published: 7 September 2021

Publisher's Note: MDPI stays neutra with regard to jurisdictional claims in published maps and institutional affiliations.

Copyright: (c) 2021 by the authors. Licensee MDPI, Basel, Switzerland. This article is an open access article distributed under the terms and conditions of the Creative Commons Attribution (CC BY) license (https:/ / creativecommons.org/licenses/by/ $4.0 /)$.
1 Department of Physical \& Numerical Science, Qurtuba University of Science \& Information Technology, Peshawar 25000, Pakistan; khola.anwer.shah@gmail.com

2 Department of Computer Science, Abbottabad University of Science and Technology, Abbottabad 22500, Pakistan

3 Department of Information Technology, The University of Haripur, Haripur 22620, Pakistan; yousaf@uoh.edu.pk

4 Pattern Recognition and Machine Learning Lab, Department of Software, Gachon University, Seongnam 13557, Korea

5 Faculty of Computing, Lahore Campus, Riphah School of Computing and Innovation, Riphah International University, Lahore 54000, Pakistan

6 Department of Computer Science, University of Buner, Buner 19290, Pakistan; inayat@ubuner.edu.pk

7 Industrial Engineering Department, College of Engineering, King Saud University, P.O. Box 800, Riyadh 11421, Saudi Arabia

8 Industrial Engineering Department, King Saud University, P.O. Box 800, Riyadh 11421, Saudi Arabia; aesayed@ksu.edu.sa

9 School of Science, College of Science, Technology, Engineering, Mathematics, RMIT University, GPO Box 2476, Melbourne, VIC 3001, Australia; mali.abdollahain@rmit.edu.au

* Correspondence: tajuom@gmail.com (T.R.); asimzeb1@gmail.com (A.Z.); adnan@gachon.ac.kr or adnan.khan@riphah.edu.pk (M.A.K.); ashafiq@ksu.edu.sa (S.A.)

Abstract: A Long-Range Wide Area Network (LoRaWAN) is one of the most efficient technologies and is widely adopted for the Internet of Things (IoT) applications. The IoT consists of massive End Devices (EDs) deployed over large geographical areas, forming a large environment. LoRaWAN uses an Adaptive Data Rate (ADR), targeting static EDs. However, the ADR is affected when the channel conditions between ED and Gateway (GW) are unstable due to shadowing, fading, and mobility. Such a condition causes massive packet loss, which increases the convergence time of the ADR. Therefore, we address the convergence time issue and propose a novel ADR at the network side to lower packet losses. The proposed ADR is evaluated through extensive simulation. The results show an enhanced convergence time compared to the state-of-the-art ADR method by reducing the packet losses and retransmission under dynamic mobile LoRaWAN network.

Keywords: adaptive data rate; convergence time; energy consumption; Internet of Things; interference; LoRaWAN; mobility; retransmissions; resource allocation

\section{Introduction}

Low Power Wide Area Networks (LPWANs) are one of the widely adopted technologies for the Internet of Things (IoT). They offer long-range and multi-year battery life with low cost solutions. Among LPWANs technologies, Narrowband (NB)-IoT, Sigfox, and Long-Range Wide Area Network (LoRaWAN) are the leading and widely adopted for static and mobile-IoT applications. Compared to NB-IoT and Sigfox, LoRaWAN offers low-cost and adaptive solutions, where the key advantages of LoRaWAN are shown in Table 1. 
Table 1. LoRaWAN Advantages Compared with NB-IoT and Sigfox [1-3].

\begin{tabular}{cccc}
\hline Features & NB-IoT & Sigfox & LoRaWAN \\
\hline Spectrum & Licensed $(>\$ 500$ million $/ \mathrm{MHz})$ & Unlicensed & Unlicensed \\
Modulation & QPSK & BPSK & chirp spread spectrum \\
Power consumption & High & Medium & Low \\
Cost High & $(\$ 15,000 /$ base station $)$ & $<\$ 5$ & Low $(\$ 100$ to $\$ 1000 / G W)$ \\
Peak current & $120-300 \mathrm{~mA}$ & $49 \mathrm{~mA}$ & $32 \mathrm{~mA}$ \\
Sleep current & $5 \mu \mathrm{A}$ & $1.44 \times 0.001$ & $1 \mu \mathrm{A}$ \\
Battery Life & $10+$ years & $2+$ years & $15+$ years \\
Bandwidth & $180 \mathrm{kHz}$ & $100 \mathrm{~Hz}$ & $125 / 250 \mathrm{kHz}$ \\
Latency & $<10 \mathrm{~s}$ & $1-30 \mathrm{~s}$ & device class dependent \\
Adaptive data rate & No & No & Yes \\
\hline
\end{tabular}

The basic LoRaWAN network design is comprised of End Devices (EDs), a Gateway (GW), and a Network Server (NS), as highlighted in Figure 1. The EDs in the LoRaWAN network interact with the environment and form the most significant part of a LoRaWAN. The GW forms a bridge between the ED and the NS. At the same time, the NS is responsible for Downlink (DL) notifications (acknowledgements) and controls the Spreading Factor (SF) and Transmit Power (TP) of ED through the Adaptive Data Rate (ADR) using Media Access Control (MAC) commands. LoRaWAN comprises three kinds of devices (Class A, $B$, and C) to serve diverse applications. Class A devices are energy-constrained and always initiate a packet in the Uplink (UL) direction using different SFs (i.e., 7 to 12). After each UL, every ED opens a Receive Window (RX1) to get a DL Acknowledgement (ACK) using the same SF and channel. If ED misses an ACK in RX1, it opens another RX2 with SF 12 using a dedicated channel $869.525 \mathrm{MHz}$. Class B end devices use beacons to interact with the GW [4]. Finally, class C end devices are not energy-efficient than class A and B because class $C$ end devices always listen to the channel.

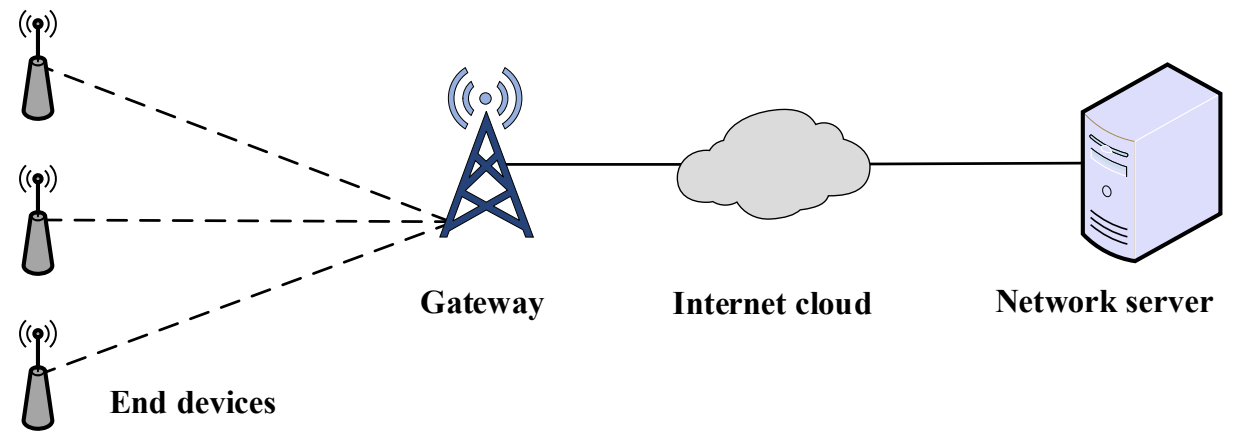

Figure 1. Star-of-Stars Topology of the LoRaWAN Network.

In a LoRaWAN network, resources (e.g., SF and TP) are managed using an adaptive method called the ADR [5]. The ADR method is implemented both at ED- and NS-sides. EDside ADR is responsible for regaining connectivity for the ED during the communication by stepping up one SF at a time. While the NS-side ADR controls both parameters, the SF and TP can efficiently maintain the battery life of the ED. However, the underlying ADR of LoRaWAN is suggested for the static EDs [6] and performs inefficiently when the EDs are mobile due to the variation in the signal strength caused by the ED movement, resulting in low Packet Delivery Ratio (PDR) and convergence time issues. This situation occurs when EDs try to retransmit a lost packet, leading to massive interference. To address the packet loss and convergence time issues, we propose an NS-side ADR and claim to make the following contributions: 
1. In the proposed work, NS-side ADR uses Gaussian-filter to smooth the SNR and receives power and allocates the resource (e.g., SF and TP) based on these parameters, resulting in a low packet loss regarding sensitivity at the GW.

2. The proposed NS-side ADR significantly increases PDR and decreases convergence time by adapting itself to the variable channels condition and allocating a suitable SF and TP.

3. The proposed NS-side ADR method can efficiently reduce energy consumption by lowering the impact of retransmission because retransmission of a packet with high parameters (e.g., SF and TP) can negatively influence the energy consumption due to high Time-On-Air (ToA).

The remaining of this paper is structured as follows: Section 2 emphasizes current state-of-the-art approaches to allocating resources to EDs. Section 3 describes the network model utilized in this paper. Section 4 presents the proposed solution. Section 5 outlines the simulation analysis of the proposed method with the state-of-the-art techniques, while Section 6 concludes this paper.

\section{Related Works}

This section highlights the existing enhancements made to the ADR, which either enhanced the PDR or the convergence time.

\subsection{Performance Improvement of the $A D R$}

To improve typical ADR efficiency, the authors in [7] averaged the Signal-to-noise ratio (SNR) of the last $P$ packets (i.e., $P=20$ ) received at the NS and then took the Standard Deviation of the resultant value. Their proposed method effectively determines the SF and TP based on the computed value using Standard Deviation. Therefore, the results of their approach indicate improved ADR performance in terms of energy consumption. However, the proposed work in [7] does not consider the convergence time, which is dependent on the channel conditions [8]. A similar approach termed ADR+ was presented in [9] by averaging the SNR of $P$ packets at the NS. Their simulation results based on OMNET++ showed improved Packet Delivery Ratio (PDR) and energy consumption outcomes. However, the ADR+ [9] failed to consider the shadowing and interference impact. Another ADR under the mobility environment was presented in [10] using the trilateration method. Their proposed work assumed a pre-defined trajectory. The authors evaluated their proposed method with ADR and showed improved performance in ToA and energy depletion. The ADR of LoRaWAN uses a fixed SF 12 during the deployment. Therefore, it suffers a massive packet loss due to interference. The authors proposed an Improved ADR (IADR) [11] to address the initial SF 12 problem in ADR. IADR aimed to assign all SFs concerning the received signal strength between the ED and GW during the network deployment. The IADR method [11] when compared to the ADR, improved the PDR. The performance results of the proposed scheme with the ADR show an improved packet delivery ratio. The authors also highlight the convergence time issue in their work. However, it failed to present a solution and resolve the convergence time issue.

The network server-based Best Equal LoRa (BE-LoRa) was proposed in [12] to optimize the energy consumption by efficiently allocating SF and TP based on optimum SINR. Their proposed method reduced energy consumption up to $36 \%$ and PDR by $17.44 \%$ compared to the typical ADR of LoRaWAN. Furthermore, another optimal energy allocation mechanism was proposed in [13] based on the ED sensing capabilities, and it showed improved energy consumption results compared to the typical ADR. The energy consumption ED can be further enhanced by utilizing a reinforcement learning method [14]. The primary aim of [14] is to allocate the best SF and TP to reduce the energy consumption and increase the PDR. Their method showed enhanced results in terms of energy consumption and PDR. 


\subsection{Performance Improvement of the Convergence Time of $A D R$}

The convergence time issue is highlighted in $[3,5,11]$ and resolved by the authors in $[15,16]$. Initially, the convergence time issue of the ADR was highlighted in [5] using various channel conditions. The authors in [5] showed through results that the ADR has the issue of convergence time due to poor adaptation of the SF and TP parameters under different underlying link conditions. In particular, their results revealed that $A D R \_A C K \_D E L A Y$ (set to 32 packets at the ED-side ADR) was mainly responsible for causing the convergence time (unconfirmed mode). However, authors in [3] claimed that ADR in unconfirmed mode does not have a convergence time issue. Furthermore, their simulation results in [3] reported that the convergence time is caused by the number of EDs and UL intervals. However, authors in $[3,5]$ failed to propose a convergence time solution.

Recently, it was realized in [15] that convergence time is a prime issue in the ADR, which reduces the PDR and needed immediate attention. Therefore, authors in [15] proposed enhanced ADR (EADR) both at ED- and NS-sides. The NS-side ADR handles their ED-side method by taking the PDR of the packets since the last changed data rate. The NS-side ADR is simple, which reduces the $P$ packet to five (i.e., $P=5$ was set) to find a suitable SF and TP. Their proposed method results show enhanced convergence time along with low energy consumption. However, the authors in [15] only considered a static environment. The convergence time issue was resolved in mobility environments in [16]. Therefore, the authors in [16] proposed two NS-side ADRs (exponentiation moving average and Gaussian-filer-based). The underlying propagation model does not change in a static environment, and the packets received at the NS with similar SNR. However, in a mobility scenario, packets are received with different SNR. Therefore, exponentiation moving average and Gaussian-filer-based approaches significantly enhanced the PDR and convergence time. Furthermore, a summary of these existing schemes is highlighted in Table 2.

Table 2. Summary of existing literature.

\begin{tabular}{cccc}
\hline Year and Ref. & ADR Type & Parameters Adjustment & Aims \\
\hline $2018-[7]$ & NS & SF and TP & To improve the energy consumption \\
$2018-[9]$ & NS & SF and TP & To improve the energy consumption and PDR \\
$2019-[10]$ & NS & SF and TP & To lower the time-on-air and improve PDR \\
$2020-[11]$ & ED & SF & To improve the PDR \\
$2021-[12]$ & NS & SF and TP & To enhance the energy consumption \\
$2021-[13]$ & NS & SF & To enhance the energy consumption \\
$2021-[14]$ & NS & SF and TP & To enhance the energy consumption \\
$2018-[5]$ & NS & SF and TP & Analysis of convergence period only \\
$2020-[3]$ & ED & SF & To improve PDR and energy consumption \\
$2020-[15]$ & ED and NS & SF and TP & To reduce the convergence period \\
$2020-[16]$ & ED and NS & SF and TP & Convergence, PDR, and energy consumption \\
Proposed ADR & NS & SF and TP & Convergence, PDR, and energy consumption \\
\hline
\end{tabular}

This paper proposes an NS-side ADR, which efficiently allocate resources (i.e., SF and $\mathrm{TP})$ to EDs based on the Gaussian filter in conjunction with the received power. The proposed ADR method efficiently enhances the convergence period, PDR, and energy consumption by reducing the packet retransmission and packet loss ratios.

\section{Network Model}

This paper assumes class $A$ end devices using the confirmed mode of communication under mobility conditions. EDs in the confirmed mode always expect a DL message (ACK) from the NS in the two receive windows. These EDs follow European region frequency with three default channels and duty cycle of $1 \%$ and $10 \%$ for UL and DL, respectively. 
In the rest of this section, we highlight link measurement and link performance models of the LoRaWAN.

\subsection{Model for the Link-Measurement}

The link measurement model considers propagation loss, fading, and shodowing models, as shown in Equation (1) [17].

$$
P_{r x}=\frac{P_{t x} \times G_{E D} \times G_{G W}}{P L} \times e^{\xi} .
$$

Equation (1) can be used to calculate the received power $\left(P_{r x}\right)$ at the GW, where $G_{E D}$ and $G_{G W}$ represent the antenna gains for the ED and GW, respectively, $P L$ is the path loss model, and $\xi$ is the shadowing term.

Log-Distance Path Loss Model

Macrocell propagation loss model (log-distance) for urban and suburban scenarios containing buildings of approximately uniform height is presented in [18].

$$
P L_{[d B]}=40 \times\left(1-4 \times 10^{-3} \times h\right) \times \log _{10}(d)-18 \times \log _{10}(h)+21 \times \log _{10}(f)+80[d B],
$$

where $d$ represents the distance in a kilometer, $h$ represents the height of the GW antenna in meters (usually measured from rooftop level), and $f$ is the frequency in $\mathrm{MHz}$.

In this work, we utilize frequency $868 \mathrm{MHz}$; therefore, for the GW antenna height (i.e., $15 \mathrm{~m}$ assumed), the $P L_{[d B]}$ is [19]

$$
P L_{[d B]}=120.5+37.6 \times \log _{10}(d) .
$$

After the $P L_{[d B]}$ is computed, log-normally distributed shadowing with a standard deviation of $10 \mathrm{~dB}$ can be added [17]. Log-normally distributed shadowing is used when there is a shadowing consequence; otherwise, it is zero.

\subsection{Model for Link Performance}

The model for the link performance is based on the probability of correct reception of a packet at lower complexity, given the earlier calculated link measurement, the amount of interference, and other system-level consequences.

\subsubsection{Receiver Sensitivity}

Successful packet reception at the GW is mainly based on the receiver sensitivity of the GW for the corresponding SF. Table 3 presents the $\operatorname{ED}\left(S_{e}\right)$ and GW $\left(S_{g}\right)$ sensitivities for each $\mathrm{SF}$, where the sensitivity of each SF increases, and the GW achieves a better sensitivity than EDs and, therefore, can decode weaker signals. A packet can be detected and successfully received by the GW if its $P_{r x}$ is above the sensitivity level. The Receiver Sensitivity $(R S)$ is significantly dependent on the choice of SF and ED uses to transmit a packet, which is given by

$$
R S=-174+10 \times \log _{10}(B W)+N F+S N R[d B],
$$

where -174 is the thermal noise computed in $\mathrm{dBm}$ for $1 \mathrm{~Hz}$ of bandwidth (BW), NF represents the Noise Factor (i.e., margin) at GW (which is set to $6 \mathrm{~dB}$ in this work) [16], SNR is the signal to noise ratio for a given SF, as mentioned in Table 3, where it can be seen that higher values of $\mathrm{SF}$ allow for better sensitivity. 
Table 3. Sensitivity and Required SNR of EDs and GW with $125-\mathrm{kHz}$ mode $[16,20,21]$.

\begin{tabular}{cccc}
\hline SF & GW Sensitivity $\left(\boldsymbol{S}_{\boldsymbol{g}}\right)[\mathrm{dBm}]$ & ED Sensitivity $\left(\boldsymbol{S}_{\boldsymbol{e}}\right)[\mathrm{dBm}]$ & SNR $[\mathrm{dB}]$ \\
\hline 12 & -142.5 & -137.0 & -20 \\
11 & -140.0 & -135.0 & -17.5 \\
10 & -137.5 & -133.0 & -15 \\
9 & -135.0 & -130.0 & -12.5 \\
8 & -132.5 & -127.0 & -10 \\
7 & -130.0 & -124.0 & -7.5 \\
\hline
\end{tabular}

\subsubsection{Interference Model}

In LoRaWAN, EDs use ALOHA to access the channel, which causes a collision when multiple EDs select the same channel for a packet transmission simultaneously. Collision in LoRaWAN is classified as when two packets collide over the same channel using the Intra-SF (collision at the GW occurs between the two packets when transmitted with the same SF and channel) or Inter-SF (collision at the GW occurs between the two packets when transmitted with different SF and same channel) interferences [17,22]. In this work, we consider both interferences impact on the dynamic LoRaWAN network, which is based on the model presented in $[17,23,24]$. According to the interference models presented in $[17,23,24]$, a packet received at the GW using $S F_{(i, j)}$ is successful, if the cumulative interference power $(\gamma)$ is higher than $\beta_{(i, j)}$ (a threshold value, as shown in Equation (5)).

$$
\beta_{(i, j)}=\left[\begin{array}{cccccc}
6 & -16 & -18 & -19 & -19 & -19 \\
-24 & 6 & -20 & -22 & -22 & -22 \\
-27 & -27 & 6 & -23 & -25 & -25 \\
-30 & -30 & -30 & 6 & -26 & -28 \\
-33 & -33 & -33 & -33 & 6 & -29 \\
-36 & -36 & -36 & -36 & -36 & 6
\end{array}\right]
$$

\section{Proposed ADR Scheme}

This section presents the proposed algorithms, running at the NS-side, as highlighted in Figure 2 and Algorithms 1 and 2. These algorithms are discussed in the remaining part of this section. Note that the working of the ED-side algorithm remains the same. Further, the symbols used in the proposed ADR are highlighted in Table 4.

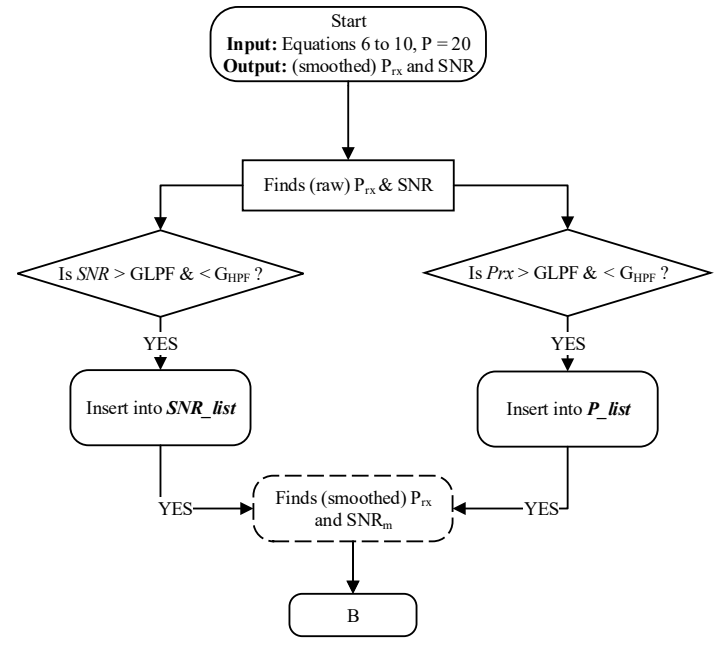

(a)

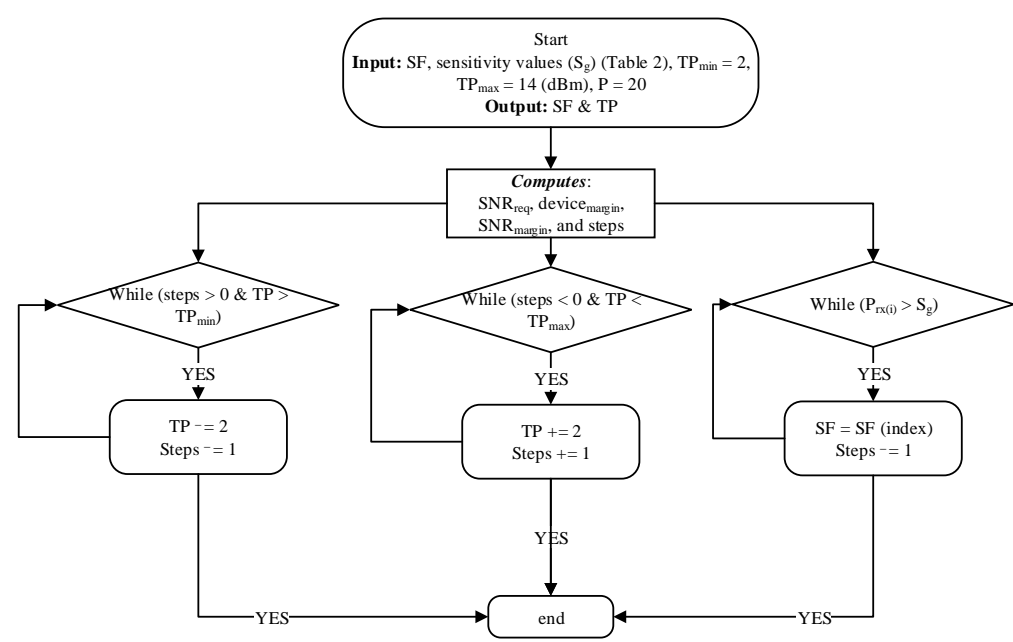

(b)

Figure 2. Flow Diagram of the Proposed ADR: (a) smoothing of $P_{r x}$ and SNR and (b) SF and TP assignment at NS side. 


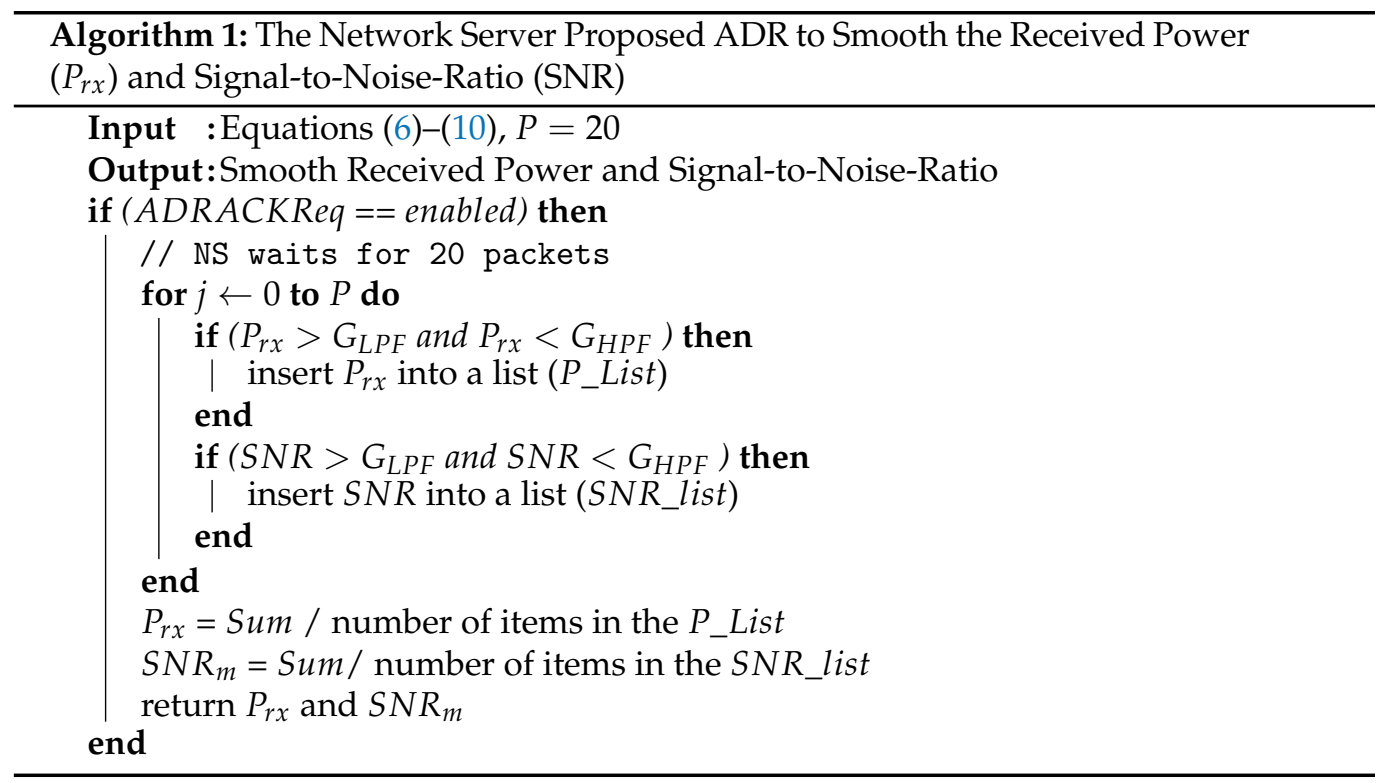

Table 4. Symbols used in the Proposed ADR.

\begin{tabular}{cc}
\hline Symbol & Description \\
\hline ADRACKReq & MAC command used by the ED to request NS for ACK $(0=$ disabled; $1=$ enabled $)$ \\
$G_{L P F}$ & Gaussian low pass filter $\left(G_{L P F}=\mu-\sigma\right)$ \\
$G_{H P F}$ & Gaussian high pass filter $\left(G_{H P F}=\mu+\sigma\right)$ \\
$P_{r x}$ & Received power at the NS associated with each packet \\
$\bar{x}$ & Mean of the $P_{r x}$ \\
$P_{-}$List & A list containing $P_{r x}$ values after filtering using Gaussian filter \\
SNR_list & A list containing $S N R$ values after filtering using Gaussian filter \\
SNR $R_{r e q}$ & Minimum SNR value required of each SF, as mentioned in Table 3 \\
$S_{g}$ & GW sensitivity value, as mentioned in Table 3 \\
steps & A number of times the ADR will be executed \\
LinkADRReq & MAC command containing SF and TP sent to ED by the NS \\
\hline
\end{tabular}

\subsection{The Proposed ADR to Smooth the Received Power $\left(P_{r x}\right)$ and $S N R$}

The proposed ADR to smooth SNR is executed when it receives $P$ packets from a corresponding ED with $A D R A C K R e q$ being enabled. To smooth the SNR, the proposed ADR computes the Sum, Mean $(\bar{x})$, Standard Deviation $(\sigma)$, Low Pass Filter $\left(G_{L P F}\right)$, and High Pass Filter $\left(G_{H P F}\right)$ using Equations (6)-(10), respectively, as under:

$$
\begin{gathered}
\text { sum }=\sum_{x=1}^{P} x_{i}, \\
\bar{x}=\frac{\text { sum }}{P}, \\
\sigma=\sqrt{\frac{1}{P-1} \sum_{i=1}^{P}\left(x_{i}-\bar{x}\right)^{2},} \\
G_{\text {LPF }}=\bar{x}-\sigma, \\
G_{H P F}=\bar{x}+\sigma,
\end{gathered}
$$

wherein Equation (8), $x_{i}$ represents a number of UL packets received at the NS, $P$ is the total number of packets (i.e., 20) required to execute the proposed algorithm. The primary purpose of Algorithm 1 is to smooth the received power $\left(P_{r x}\right)$ and SNR values associated 
with each UL packet received by the NS. To smooth the signal variation (i.e., in $P_{r x}$ ) caused due to mobility during the ED movement, the proposed ADR compares the computed $P_{r x}$ to Gaussian filters (i.e., $G_{L P F}$ and $G_{H P F}$ ). The resultant values containing $P_{r x}$ are recorded in a list $\left(P_{\text {list }}\right)$. Next, to smooth the SNR values, these are compared to $G_{L P F}$ and $G_{H P F}$ (these are computed for SNR values separately) and saved in a list $S N R_{\text {list }}$. Finally, smoothed $P_{r x}$ and SNR $\left(S N R_{m}\right)$ are computed. These are further utilized in Algorithm 2.

\subsection{The Network Server Proposed ADR Method}

The proposed ADR in Algorithm 2 utilizes the default ADR settings, such as computing the $S N R_{\text {reg }}, S N R_{\text {margin }}$, and steps. Based on the smoothed $P_{r x}$ and $S N R m$ values, the proposed ADR finds a suitable SF concerning the GW sensitivity values $\left(S_{g}\right)$ to lower the chances of packet loss arriving at GW under the sensitivity. Then by using steps, the proposed ADR finds the best possible TP for the concerned ED. If steps has zero value, it shows the ADR is already using the best value of TP. When steps is higher than zero, it shows that the current TP is not optimized (i.e., consumes more energy). Therefore, the proposed ADR decreases the TP by 2 (until it reaches the minimum limit) to optimize energy consumption (i.e., lower energy consumption). On the other hand, when steps is lower than zero, the proposed ADR increases the TP by 2 (until it reaches the maxim value). When both parameters (i.e., $\mathrm{SF}$ and $\mathrm{TP}$ ) are found, these are communicated to the concerned ED using a MAC command LinkADRReq.

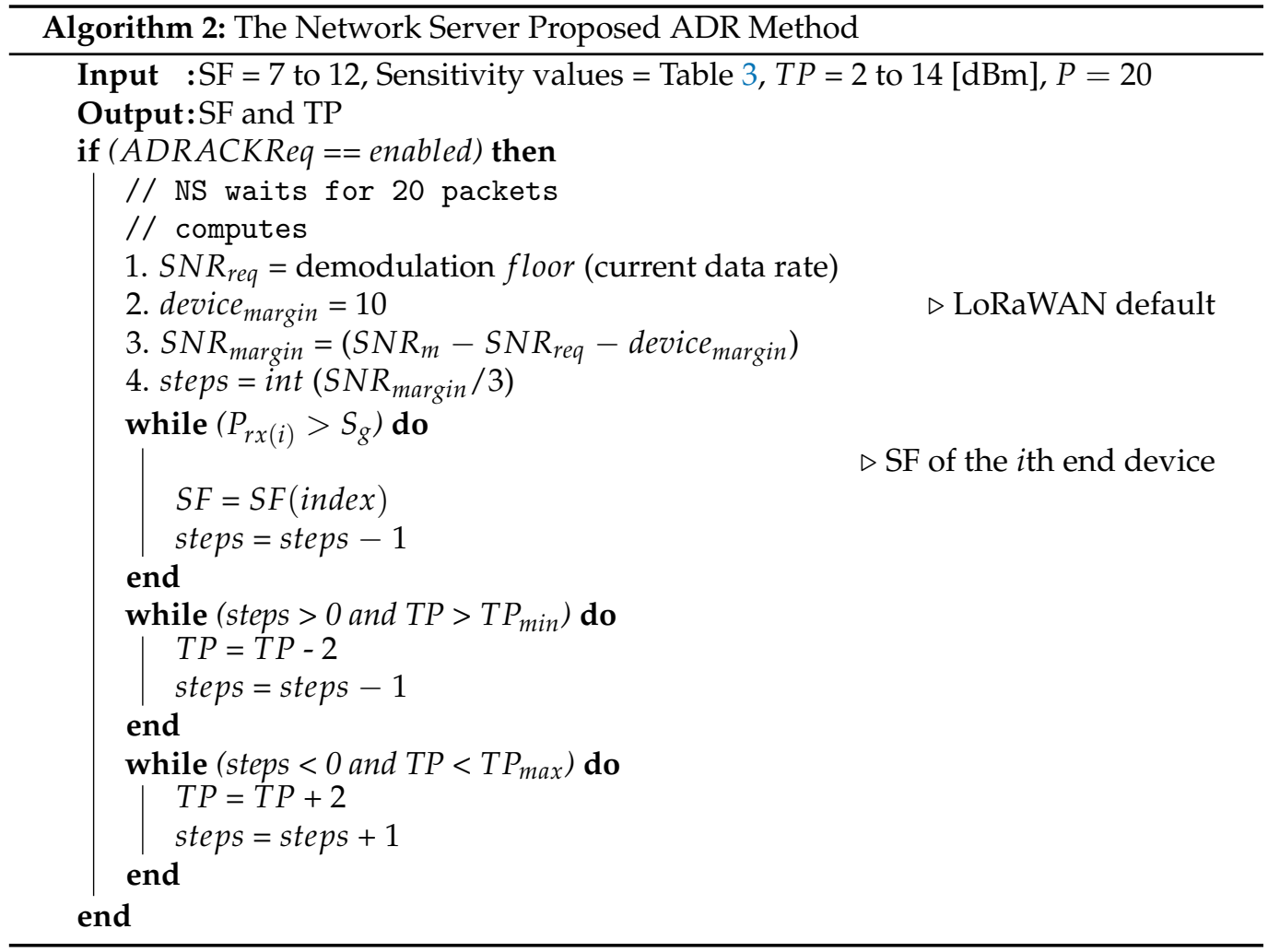

\section{Performance Analysis of the Proposed ADR}

This section describes the performance evaluation of the proposed ADR in comparison with ADR. The performance is evaluated in terms of PDR, convergence time, and energy consumption under a mobility environment using a confirmed communication mode. The performance evaluation is done using NS-3 [25]. 


\subsection{Application Scenario}

In this work, we consider a pet-tracking IoT-enabled application with various requirements, such as packet length, reliability, and mobility [26-28]. Every ED in the simulation transmits 48 packets/day with a packet size of 50 bytes.

\subsection{Simulation Environment}

We consider class $A$ EDs $(\mathrm{N})$ uniformly distributed around a single GW within a 5-km radius. The GW and ED antenna heights are set to 15 and $1.5 \mathrm{~m}$, respectively. The rest of the simulation parameters are presented in Table 5.

Table 5. Simulation Parameters.

\begin{tabular}{cc}
\hline Parameter & Value \\
\hline Simulation time $[\mathrm{h}]$ & 24 \\
GW & 1 \\
Uplink interval & 48 packets/day \\
Packet size [bytes] & $50[23]$ \\
Total number of transmission allowed & 8 \\
Path loss exponent & $3.76[19]$ \\
Underlying propagation model & log-distance \\
Shadowing & de-correlation distance $=110 \mathrm{~m}[26]$ and \\
variance $=6 \mathrm{~dB}[29]$ \\
Mobility model & random walk 2-D $([3,16])$ \\
ED movement speed [m/s] & $0.5 \sim 1.5([3,16])$ \\
Transmit power [dBm] & $2 \sim 14$ \\
Frequency region & EU-868 \\
\hline
\end{tabular}

\subsection{Performance Analysis}

\subsubsection{Packet Delivery Ratio}

In this work, the Packet Delivery Ratio (PDR) is defined as when ED receives a DL ACK from the NS in one of the eight transmission attempts [30]. Figure 3 shows the PDR of the proposed ADR and the ADR for different number of ED under mobility conditions. The PDR trend shown in Figure 3 decreases with an increasing number of EDs. When the number of EDs increase, the UL traffic is increased, which cause a bottleneck at the GW, resulting in packet loss due to interference and packets arriving under sensitivity in the case of ADR. However, in the proposed ADR, the SF and TP are adapted based on the smoothed signal strength and $P_{r x}$, resulting in improved PDR.

\subsubsection{Convergence Time}

The convergence time in LoRaWAN is defined as the time taken by ED to reach a steady SF, TP, and PDR. The convergence time occurs after the initial deployment phase, and it can last for hours or even days and is mainly dependent on the number of EDs and uplink period $[5,11,15,16]$. At the ED-side ADR, this convergence time is mainly based on both $A D R \_A C K \_L I M I T$ and $A D R \_A C K \_D E L A Y[5,15]$. This time is affected by the arbitrary uplink history used at the NS (20 packets) on the NS-side. Before adopting SF or $\mathrm{TP}$, the NS-side ADR must wait for the 20 packets from each ED at the NS. This waiting time increases the convergence time, and this increases the massive packet loss.

In Figure 4, ADR suffers from high convergence time because it cannot adapt itself to the variable channel condition. The new parameters (i.e., SF and TP) identified by the ADR could not successfully deliver a packet to the GW due to the underlying propagation environment caused by end device mobility. Therefore, NS cannot identify a suitable SF, resulting in massive packet loss at GW due to arriving under the sensitivity. In addition, the uplink traffic is increased from the end devices involved in the communication due to the packet loss. As a result, this increased uplink traffic causes a bottleneck at the GW, 
resulting in a large number of packet losses due to interference. Therefore, during the $24 \mathrm{~h}$ of simulation time, ADR failed to converge to a stable PDR due to end device mobility, which causes interference.

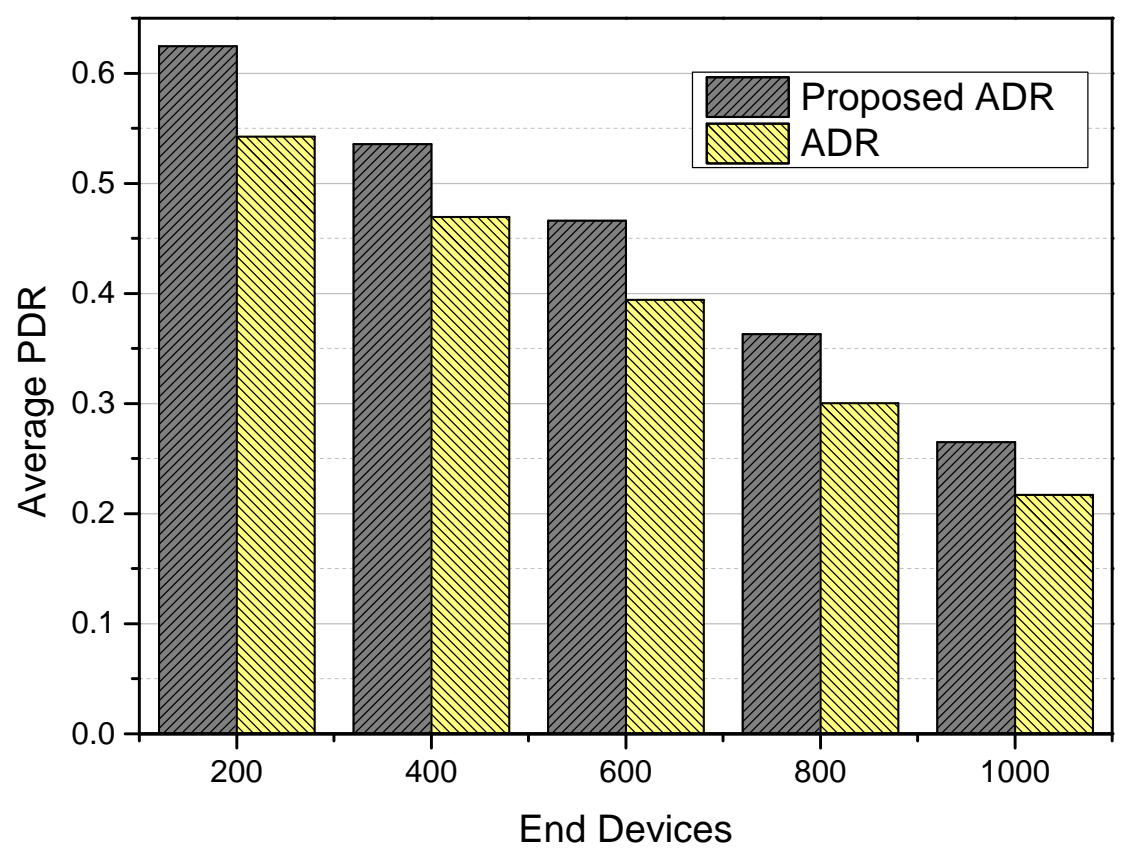

Figure 3. Average Packet Delivery Ratio of the Proposed ADR and ADR.

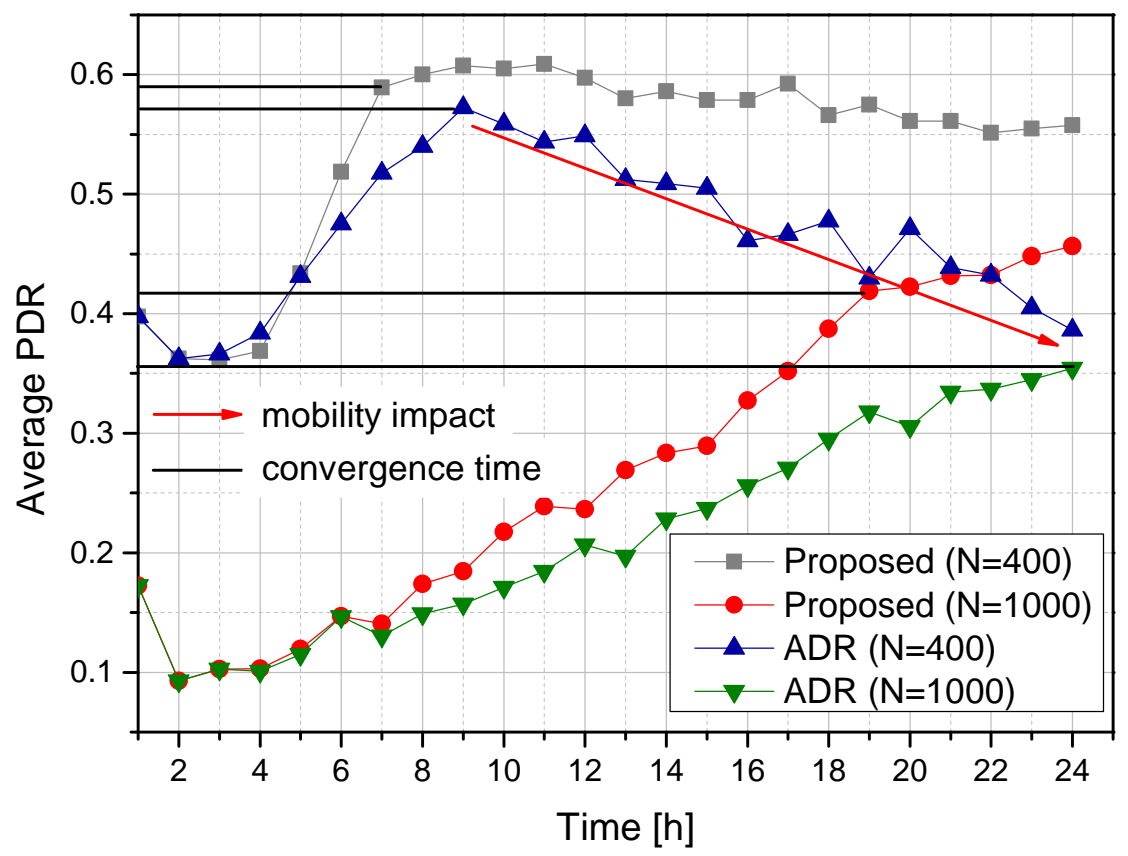

Figure 4. Convergence time and PDR of the Proposed ADR and ADR.

However, in the proposed ADR for the $\mathrm{N}=400$ and $\mathrm{N}=1000$, the convergence time is 9 and $19 \mathrm{~h}$, respectively. The primary reason behind this low convergence time is that the proposed ADR at the NS side manages to smooth the signal strength and identify the best possible configuration containing both SF and TP. In addition, the proposed ADR considers the $P_{r x}$ value, which helps to identify a suitable SF for the ED, thus decreasing the chances of packet loss arriving under the SF sensitivity thresholds. 


\subsubsection{Energy Consumption}

The energy consumption is determined by dividing the overall energy spent by EDs during the simulation period by the number of packets successfully received [31,32]. We used the energy consumer module from [33] and the Semtech SX1272/73 datasheet with a $3.3 \mathrm{~V}$ supply voltage [21] in this paper.

Figure 5 shows that energy consumption increases for both the proposed ADR and the ADR when the number of EDs increases. This is because both proposed ADR and ADR utilize SF = 12 during the initial network deployment, which causes significant interference due to high ToA. In addition, the energy consumption of the ADR is relatively higher than the proposed ADR due to the number of retransmission. When a packet is lost in the confirmed mode, it is retransmitted up to 7 times. This retransmission causes significant energy consumption in the case of ADR. Therefore, we believe that this high energy consumption in ADR is caused due to the high SF, TP, ToA, and retransmission [31]. However, in the proposed ADR, the energy consumption is low because it assigns a suitable $\mathrm{SF}$ and TP using the Gaussian filter and $P_{r x}$. This appropriate SF and TP adaptation leads to better PDR, resulting in lowering the number of retransmissions. Therefore, the proposed ADR's energy consumption is lower than ADR, outperforming the ADR.

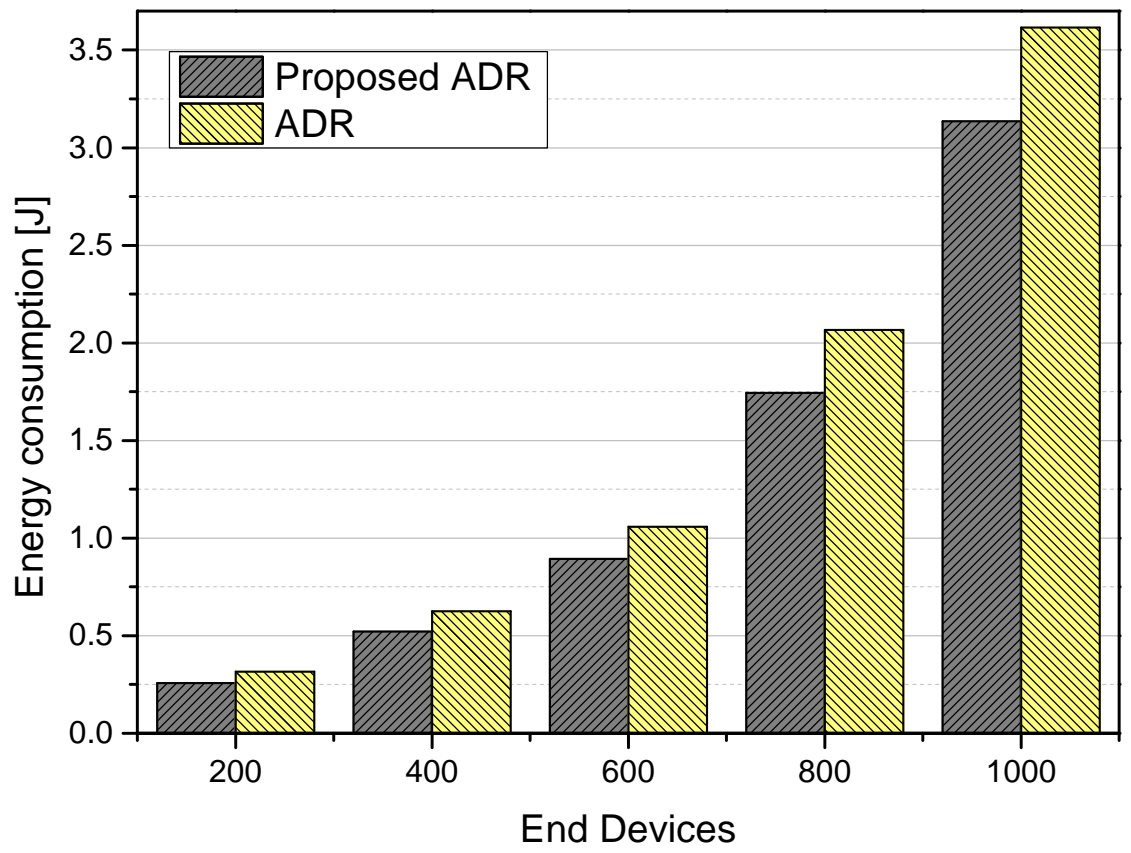

Figure 5. Average Energy Consumption in Joules for the Proposed ADR and ADR.

\subsubsection{Overall Performance}

Figure 6 shows the fraction of the PDR and Packet Loss Ratio (PLR) caused by interference (PLR-I), receiver saturation (PLR-R), under the sensitivity (PLR-S), and transmission of ACK (PLR-T). PLR-I occurs when two or more packets overlap each using the same or different SFs over the same channel. Here, the impact of PLR-I for the proposed ADR and ADR is almost identical. However, the proposed ADR PLR-I is a little lower. PLR-R occurs when all the available reception paths at the GW are busy (there are 8 parallel reception paths at the GW for the default 3 channels). PLR-R for both of the proposed and ADR is primarily similar. PLR-R and PLR-I are subject to UL traffic. When UL traffic increases, both PLR-R and PLR-I increase due to congestion at the GW. However, a significant improvement in the case of PLR-S (it occurs when packets arrive at the GW under the required sensitivity) for the proposed ADR is observed, as shown in Figure 6a. This is because the proposed ADR allocates the SF based on the $P_{r x}$, which reduces the chances of packets arriving at the GW under the sensitivity. 


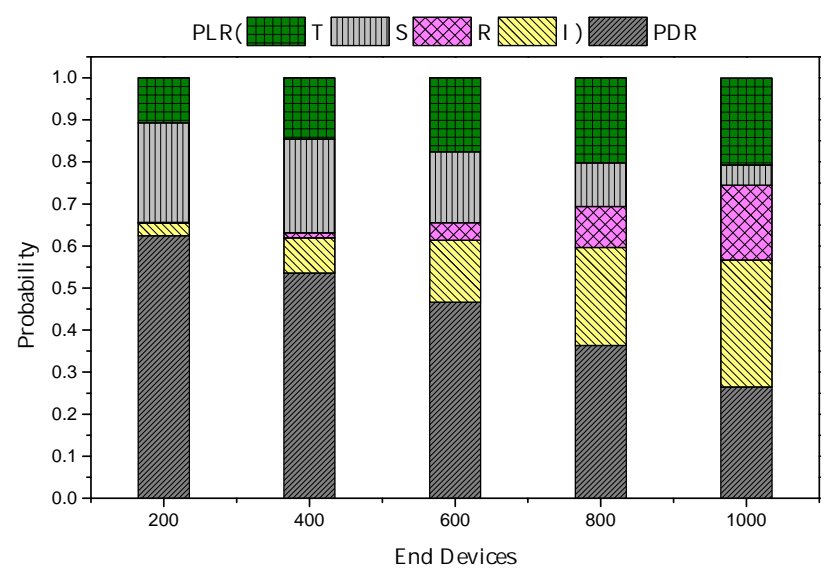

(a) Proposed ADR

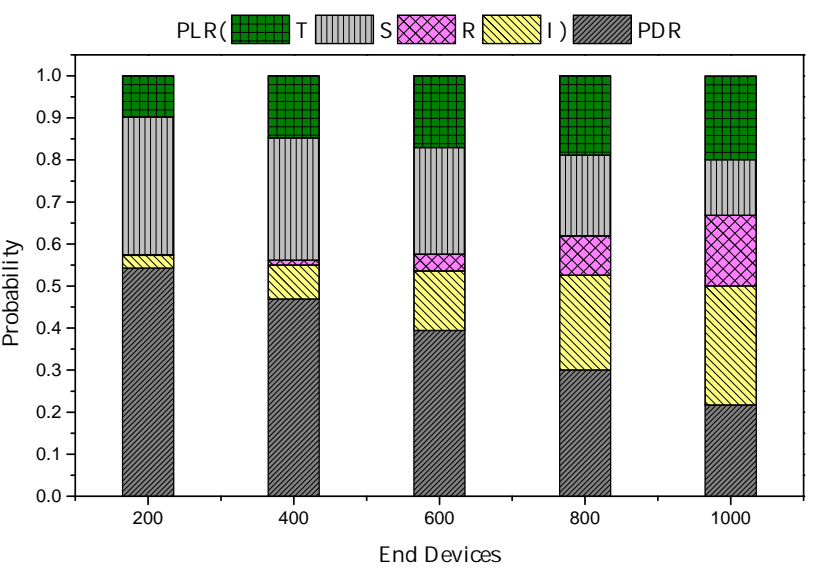

(b) ADR

Figure 6. Analysis of PDR and PLRs.

On the other hand, the PLR-S impact is high in ADR, as shown in Figure 6b. This is because ADR cannot allocate a suitable SF, which increases the chances of packets arriving at the GW under the sensitivity. Therefore, PLR-S is significant in the ADR. Another reason for the packet loss in the LoRaWAN network is the PLR-T. PLR-T occurs when GW is busy transmitting ACK packet to ED; simultaneously, a packet arriving at the GW is discarded. Thus, PLR-T impact is almost identical in both cases.

\subsubsection{Performance Improvement of the Proposed ADR}

Figure 7 shows the improvement of the proposed ADR with the ADR. The enhancement shown in Figure 7 represents the average improvement in percentage for energy, PDR, and PLRs. Thus, the proposed ADR has significantly improved the performance, particularly in energy consumption (i.e., $16 \%$ improvement) and PDR (i.e., $18 \%$ improvement). In PLRs, the proposed ADR enhances the performance by $5 \%, 39 \%, 3 \%$, and $2 \%$ for the PLR-I, PLR-S, PLR-R, and PLR-T, respectively.

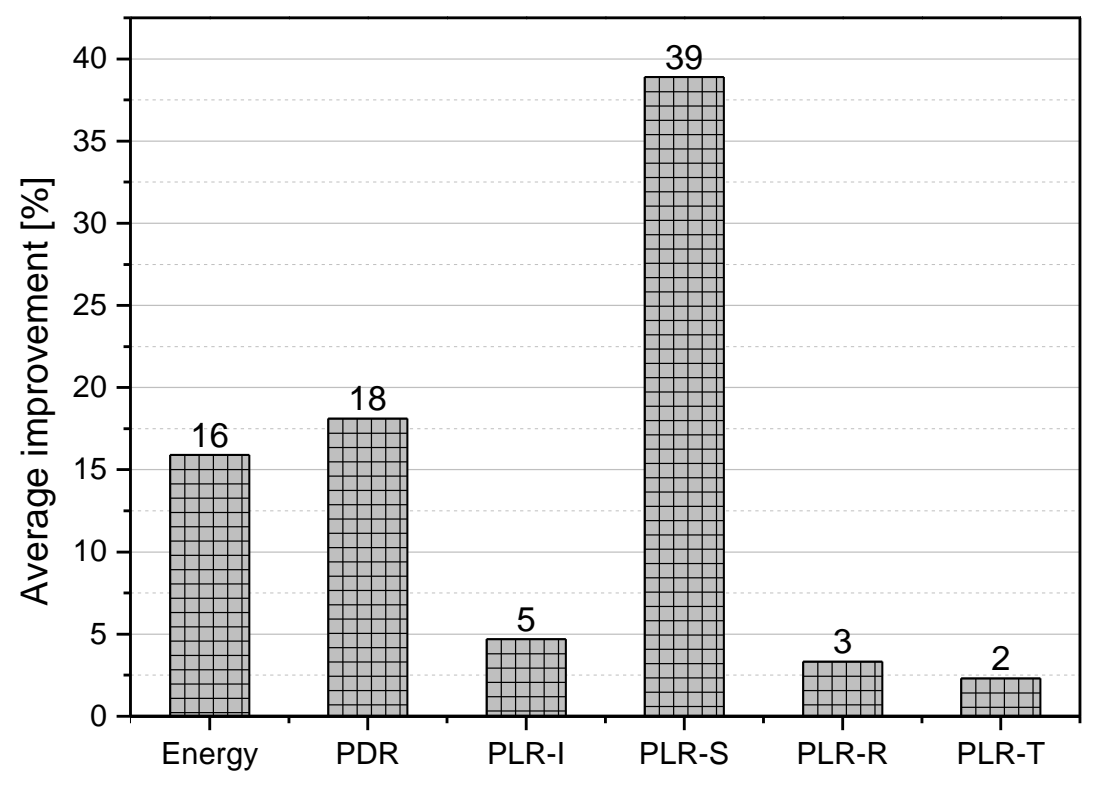

Figure 7. Performance Improvement in Percentage for the Proposed ADR. 


\section{Conclusions}

In a LoRaWAN network, resources (e.g., SF and TP) are managed using an adaptive method called the Adaptive Data Rate (ADR). The NS-side ADR of the LoRaWAN network controls both the SF and TP parameters to maintain the ED's battery life efficiently. On the other hand, ED-side ADR only increments SF to keep the complexity of ED as low as possible. However, the ADR is inefficient when the EDs are mobile due to the variation in the signal strength caused by the ED movement. Therefore, we proposed an ADR at the NSside based on the Gaussian filter and the received power, which efficiently assigned both SF and TP. The simulation results showed that the proposed ADR is efficient at reducing convergence time and energy consumption. We also observed that the proposed ADR efficiently enhances the packet delivery ratio by reducing packet losses. Furthermore, since the proposed ADR works at the NS side, it can be easily integrated at the NS as part of routine maintenance. Therefore, we believe that the proposed ADR can be adopted for mobile IoT end devices, which require low convergence time and a high packet delivery ratio with low energy consumption. In the future, we can reduce the interference impact by employing an ADR at both the ED- and NS-sides.

Author Contributions: Conceptualization, K.A.; Data curation, A.Z.; Formal analysis, T.R.; Funding acquisition, S.A., A.E.A. and M.A.; Resources, I.K.; Software, Y.S.; Writing-review \& editing, M.A.K. All authors have contributed equally. All authors have read and agreed to the published version of the manuscript.

Funding: The authors extend their appreciation to King Saud University for funding this work through Researchers Supporting Project number (RSP-2021/387), King Saud University, Riyadh, Saudi Arabia.

Institutional Review Board Statement: Not applicable.

Informed Consent Statement: Not applicable.

Data Availability Statement: The data that support the findings of this study are available from the author upon reasonable request.

Conflicts of Interest: The authors declare no conflict of interest.

\section{References}

1. Gomez, C.; Veras, J.C.; Vidal, R.; Casals, L.; Paradells, J. A sigfox energy consumption model. Sensors 2019, 19, 681. [CrossRef] [PubMed]

2. Chalapati, S. Comparison of LPWA Technologies and Realizable Use Cases. 2018. Available online: https://www.nctatechnicalpapers com/Paper/2018/2018-comparison-of-lpwa-technologies-and-realizable-use-cases (accessed on 22 August 2021).

3. Farhad, A.; Kim, D.H.; Kim, B.H.; Mohammed, A.F.Y.; Pyun, J.Y. Mobility-Aware Resource Assignment to IoT Applications in Long-Range Wide Area Networks. IEEE Access 2020, 8, 186111-186124. [CrossRef]

4. Haxhibeqiri, J.; De Poorter, E.; Moerman, I.; Hoebeke, J. A survey of lorawan for iot: From technology to application. Sensors 2018, 18, 3995. [CrossRef] [PubMed]

5. Li, S.; Raza, U.; Khan, A. How Agile is the Adaptive Data Rate Mechanism of LoRaWAN. In Proceedings of the IEEE Global Communications Conference (GLOBECOM), Abu Dhabi, United Arab Emirates, 9-13 December 2018; pp. 206-212.

6. SEMTECH. LoRaWAN Mobile Applications: Blind ADR. 2019. Available online: https://lora-developers.semtech.com/library/ tech-papers-and-guides/blind-adr/ (accessed on 21 August 2021).

7. Peruzzo, A.; Vangelista, L. A power efficient adaptive data rate algorithm for LoRaWAN networks. In Proceedings of the 2018 21st International Symposium on Wireless Personal Multimedia Communications (WPMC), Chiang Rai, Thailand, 25-28 November 2018; pp. 90-94.

8. Kousias, K.; Caso, G.; Alay, Ö.; Lemic, F. Empirical Analysis of LoRaWAN Adaptive Data Rate for Mobile Internet of Things Applications. In Proceedings of the 2019 on Wireless of the Students, by the Students, and for the Students Workshop, Los Cabos, Mexico, 21 October 2019; pp. 9-11.

9. Slabicki, M.; Premsankar, G.; Di Francesco, M. Adaptive configuration of LoRa networks for dense IoT deployments. In Proceedings of the IEEE/IFIP Network Operations and Management Symposium, Taipei, Taiwan, 23-27 April 2018; pp. 1-9.

10. Benkahla, N.; Tounsi, H.; Ye-Qiong, S.; Frikha, M. Enhanced ADR for LoRaWAN networks with mobility. In Proceedings of the 2019 15th International Wireless Communications \& Mobile Computing Conference (IWCMC), Tangier, Morocco, 24-28 June 2019; pp. 1-6. 
11. Farhad, A.; Kim, D.H.; Kwon, D.; Pyun, J.Y. An Improved Adaptive Data Rate for LoRaWAN Networks. In Proceedings of the 2020 IEEE International Conference on Consumer Electronics-Asia (ICCE-Asia), Seoul, Korea, 1-3 November 2020; pp. 1-4.

12. Al-Gumaei, Y.A.; Aslam, N.; Chen, X.; Raza, M.; Ansari, R.I. Optimising Power Allocation in LoRaWAN IoT Applications. IEEE Internet Things J. 2021. [CrossRef]

13. Gleonec, P.D.; Ardouin, J.; Gautier, M.; Berder, O. Energy Allocation for LoRaWAN Nodes with Multi-Source Energy Harvesting. Sensors 2021, 21, 2874. [CrossRef] [PubMed]

14. Khalifeh, A.; Aldahdouh, K.; Alouneh, S. LoRaWAN Energy Optimization with Security Consideration. Int. Arab J. Inf. Technol. 2021, 18, 476-483.

15. Finnegan, J.; Farrell, R.; Brown, S. Analysis and Enhancement of the LoRaWAN Adaptive Data Rate Scheme. IEEE Internet Things J. 2020, 7, 7171-7180. [CrossRef]

16. Farhad, A.; Kim, D.H.; Subedi, S.; Pyun, J.Y. Enhanced LoRaWAN Adaptive Data Rate for Mobile Internet of Things Devices. Sensors 2020, 20, 6466. [CrossRef] [PubMed]

17. Farhad, A.; Kim, D.H.; Pyun, J.Y. Resource Allocation to Massive Internet of Things in LoRaWANs. Sensors 2020, $20,2645$. [CrossRef] [PubMed]

18. ETSI. LTE; Evolved Universal Terrestrial Radio Access (E-UTRA); Radio Frequency (RF) System Scenarios (3GPP TR 36.942 Version 8.2.0 Release 8). 2009. Available online: https://www.etsi.org/deliver/etsi_tr/136900_136999/136942/08.02.00_60/tr_13 6942v080200p.pdf (accessed on 22 February 2021).

19. Farhad, A.; Kim, D.; Pyun, J. Scalability of LoRaWAN in an Urban Environment: A Simulation Study. In Proceedings of the Eleventh International Conference on Ubiquitous and Future Networks (ICUFN), Zagreb, Croatia, 2-5 July 2019 ; pp. 677-681. [CrossRef]

20. Semtech. Semtech SX1301 WIRELESS \& SENSING PRODUCTS Datasheet. 2017. Available online: https://www.semtech.com/ products/wireless-rf/lora-gateways/sx1301 (accessed on 2 January 2020).

21. Semtech. Semtech WIRELESS \& SENSING PRODUCTS, Sx1272. 2017. Available online: https://www.semtech.com/products/ wireless-rf/lora-transceivers/sx1272 (accessed on 2 January 2020).

22. Reynders, B.; Wang, Q.; Tuset-Peiro, P.; Vilajosana, X.; Pollin, S. Improving reliability and scalability of lorawans through lightweight scheduling. IEEE Internet Things J. 2018, 5, 1830-1842. [CrossRef]

23. Magrin, D.; Centenaro, M.; Vangelista, L. Performance evaluation of LoRa networks in a smart city scenario. In Proceedings of the IEEE International Conference on communications (ICC), Paris, France, 21-25 May 2017; pp. 1-7.

24. Farhad, A.; Kim, D.; Sthapit, P.; Pyun, J. Interference-Aware Spreading Factor Assignment Scheme for the Massive LoRaWAN Network. In Proceedings of the 2019 International Conference on Electronics, Information, and Communication (ICEIC), Auckland, New Zealand, 22-25 January 2019; pp. 1-2. [CrossRef]

25. Network Simulator (ns)-3. Available online: https:/ / www.nsnam.org/ (accessed on 11 December 2019).

26. 3GPP. Cellular System Support for Ulta-Low Complexity and Low Throughput Internet of Things (CIoT). 2016. Available online: https:/ /itectec.com/archive/3gpp-specification-tr-45-820/ (accessed on 4 March 2020).

27. Ducrot, N.; Ray, D.; Saadani, A.; Hersent, O.; Pop, G.; Remond, G. Lora Device Developer Guide. 2016. Available online: https:/ / developer.orange.com/wp-content/uploads/LoRa-Device-Developer-Guide-Orange.pdf (accessed on 6 May 2020).

28. Rahman, T.; Zhou, Z.; Ning, H. Energy efficient and accurate tracking and detection of continuous objects in wireless sensor networks. In Proceedings of the 2018 IEEE International Conference on Smart Internet of Things (SmartIoT), Xi'an, China, 17-19 August 2018; pp. 210-215.

29. Capuzzo, M.; Magrin, D.; Zanella, A. Confirmed traffic in LoRaWAN: Pitfalls and countermeasures. In Proceedings of the 2018 17th Annual Mediterranean Ad Hoc Networking Workshop (Med-Hoc-Net), Capri, Italy, 20-22 June 2018; pp. 1-7.

30. Magrin, D.; Capuzzo, M.; Zanella, A. A thorough study of LoRaWAN performance under different parameter settings. IEEE Internet Things J. 2019, 7, 116-127. [CrossRef]

31. Finnegan, J.; Brown, S.; Farrell, R. Modeling the Energy Consumption of LoRaWAN in ns-3 Based on Real World Measurements. In Proceedings of the 2018 Global Information Infrastructure and Networking Symposium (GIIS), Thessaloniki, Greece, 23-25 October 2018; pp. 1-4.

32. Du, X.; Zhou, Z.; Zhang, Y.; Rahman, T. Energy-efficient sensory data gathering based on compressed sensing in IoT networks. J. Cloud Comput. 2020, 9, 19. [CrossRef]

33. Bor, M.C.; Roedig, U.; Voigt, T.; Alonso, J.M. Do LoRa low-power wide-area networks scale? In Proceedings of the 19th ACM International Conference on Modeling, Analysis and Simulation of Wireless and Mobile Systems, Zagreb, Croatia, 13-17 July 2016; pp. 59-67. 